International Journal of Pure and Applied Mathematics

Volume 103 No. 3 2015, 385-394

ISSN: 1311-8080 (printed version); ISSN: 1314-3395 (on-line version)

url: http://www.ijpam.eu

doi: http://dx.doi.org/10.12732/ijpam.v103i3.1

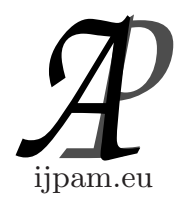

\title{
NUMERICAL INVESTIGATION OF THE HYBRID FUZZY DIFFERENTIAL EQUATIONS USING LEAPFROG METHOD
}

\author{
S. Sekar ${ }^{1}$, K. Prabhavathi ${ }^{2}$ \\ ${ }^{1}$ Department of Mathematics \\ Government Arts College (Autonomous) \\ Salem, 636 007, Tamil Nadu, INDIA \\ ${ }^{2}$ Department of Mathematics \\ Bannari Amman Institute of Technology \\ Sathyamangalam, 638 401, Tamil Nadu, INDIA
}

\begin{abstract}
In this paper, We develop the Leapfrog method for solving hybrid fuzzy differential equations (HFDE) based on the generalized concept of higherorder fuzzy differentiability [19]. The obtain discrete solutions were compared with another method taken from the literature [19]. The new method has a lower computational cost which effects the time consumption. We assume that the fuzzy function and its derivative are Hukuhara differentiable. The numerical example was given to illustrate the efficiency of the method.
\end{abstract}

AMS Subject Classification: 65L80, 65L05

Key Words: fuzzy differential equations, fuzzy initial value problems, hybrid fuzzy differential equations, single term Haar wavelet series, Leapfrog method

\section{Introduction}

A hybrid system is a dynamic system that exhibits both continuous and dis-

Received: February 8, 2015

(C) 2015 Academic Publications, Ltd.

$\S_{\text {Correspondence author }}$ url: www.acadpubl.eu 
crete dynamic behavior - a system that can both flow (described by a differential equation) and jump (described by a difference equation or control graph). Often, the term "hybrid dynamic system" is used, to distinguish over hybrid systems such as those that combine neural nets and fuzzy logic, or electrical and mechanical drive lines. A hybrid system has the benefit of encompassing a larger class of systems within its structure, allowing for more flexibility in modeling dynamic phenomena.

In general, the state of a hybrid system is defined by the values of the continuous variables and a discrete control mode. The state changes either continuously, according to a flow condition, or discretely according to a control graph. Continuous flow is permitted as long as so-called invariants hold, while discrete transitions can occur as soon as given jump conditions are satisfied. Discrete transition may be associated with events.

Fuzzy differential equations serve as mathematical models for many exciting real-world problems, not only in science and technology but also in such diverse fields as bio dynamics models [7], population models [8], and modelling hydraulic [2]. Initially, the derivative of fuzzy-valued functions was first introduced by Chang and Zadeh [3]. It was followed by Dubois and Prade [4], who used the extension principle in their approach. Other methods have been discussed by Puri and Ralescu $[15,16]$; they generalized and extended the concept of Hukuhara differentiability (-derivative) from set-valued mappings to the class of fuzzy mappings. Subsequently, using -derivative, Kaleva $[9,10]$ and Seikkala [22] developed the theory of fuzzy differential equations. In the last few years, many researchers have worked on theoretical and numerical solution of FDEs $[13,14]$, specially some authors considered the second-order fuzzy differential equations $[1,5,20]$.

Recently Sekar and his team of researchers analyzed second order linear system with singular-A, Second-order linear singular systems, first order linear fuzzy differential equations and first order linear singular systems using Leapfrog method [11, 12, 18, 21]. The objective of this article is to use the Leapfrog method to solve hybrid fuzzy differential equations (discussed by Sekar et al. [19],). Also Leapfrog method is introduced for the hybrid fuzzy differential equations. As compared to [19], our method is simpler and consumes less computer time. The paper is organized as follows: in Section 2 and 3 are devoted to the preliminaries and basic concepts of hybrid fuzzy differential equations. Section 3 we describe the Leapfrog method required for our subsequent development. In Section 4 general formation of the hybrid fuzzy differential equations presented and in Section 5 we apply the proposed numerical method to the hybrid fuzzy differential equations and report our numerical finding and 
demonstrate the accuracy of the proposed method.

\section{Preliminaries}

Denote by $E^{1}$ the set of all functions $u: R \rightarrow[0,1]$ such that (i) $\mathrm{u}$ is normal, that is, there exist an $x_{0} \in R$ such that $u\left(x_{0}\right)=1$, (ii) $\mathrm{u}$ is a fuzzy convex, that is, for $x, y \in R$ and $0 \leq \lambda \leq 1, u(\lambda x+(1 \lambda) y) \geq \min \{u(x), u(y)\}$, (iii) $\mathrm{u}$ is upper semicontinuous, and (iv) $[u]^{0} \equiv$ the closure of $\{x \in R: u(x)>0\}$ is compact. For $0<\alpha \leq 1$, we define $[u]^{\alpha}=\{x \in R: u(x) \geq \alpha\}$. An example of a $u \in E^{1}$ is given by

$$
u(x)= \begin{cases}4 x-3, & \text { if } x \in(0.75,1], \\ -2 x+3, & \text { if } x \in(1,1.5), \\ 0, & \text { if } x \notin(0.75,1.5) .\end{cases}
$$

The $\alpha$-level sets of $\mathrm{u}$ in (1) are given by $[u]^{\alpha}=[0.75+0.25 \alpha, 1.50 .5 \alpha]$. For later purpose, we define $\hat{o} \in E^{1}$ as $\hat{o}(x)=1$ if $x=0$ and $\hat{o}(x)=0$ if $x \neq 0$.

Next we review the Seikkala derivative [22] of $x: I \rightarrow E^{1}$ where $I \subset R$ is an interval. If $\left[x(t)^{a}\right]=\left[\underline{x}^{a}(t), \bar{x}^{a}(t)\right]$ for all $t \in I$ and $a \in[0,1]$, then $\left[x^{\prime}(t)^{a}\right]=\left[\underline{x}^{\prime}(t)^{a},\left(\bar{x}^{a}\right)^{\prime}(t)\right]$ if $x^{\prime}(t) \in E^{1}$. Next consider the initial value problem (IVP)

$$
u(x)=\left\{\begin{array}{l}
x^{\prime}(t)=f(t, x(t)), \\
x(0)=x_{0}
\end{array}\right.
$$

where $f:[0, \infty) \times R \rightarrow R$ is continuous. We would like to interpret (1) using the Seikkala derivative and $x_{0} \in E^{1}$. Let $\left[x_{0}\right]^{a}=\left[\underline{x}_{0}^{a}, \bar{x}_{0}^{a}\right]$ and $[x(t)]^{a}=\left[\underline{x}^{a}(t), \bar{x}^{a}(t)\right]$. By the Zadeh extension principle we get $f:[0, \infty) \times E^{1} \rightarrow E^{1}$ where $[f(t, x)]^{a}=$ $\min f(t, u): u \in\left[\underline{x}^{a}(t), \bar{x}^{a}(t)\right], \max f(t, u): u \in\left[\underline{x}^{a}(t), \bar{x}^{a}(t)\right]$. Then $x:[0, \infty) \rightarrow$ $E^{1}$ is a solution of (1) using the Seikkala derivative and $x_{0} \in E^{1}$ if

$$
\begin{aligned}
& \left(\underline{x}^{a}\right)^{\prime}(t)=\operatorname{minf}(t, u): u \in\left[\underline{x}^{a}(t), \bar{x}^{a}(t)\right], \underline{x}^{a}(0)=\underline{x}_{0}^{a}, \\
& \left(\bar{x}^{a}\right)^{\prime}(t)=\max f(t, u): u \in\left[\underline{x}^{a}(t), \bar{x}^{a}(t)\right], \bar{x}^{a}(0)=\bar{x}_{0}^{a},
\end{aligned}
$$

for all $t \in[0, \infty)$ and $a \in[0,1]$. Lastly consider an $f:[0, \infty) \times R \times R \rightarrow R$ which is continuous and the IVP

$$
\left\{\begin{array}{l}
x^{\prime}(t)=f(t, x(t), k) \\
x(0)=x_{0}
\end{array}\right.
$$


As in (1), to interpret (2) using the Seikkala derivative and $x_{0}, k \in E^{1}$, by the Zadeh extension principle we use $f:[0, \infty) \times E^{1} \times E^{1} \rightarrow E^{1}$ where

$$
\begin{gathered}
{[f(t, x, k)]^{a}=\left[\min f\left(t, u, u_{k}\right): u \in\left[\underline{x}^{a}(t), \bar{x}^{a}(t)\right], u_{k} \in\left[\underline{k}^{a}, \bar{k}^{a}\right],\right.} \\
\left.\max f\left(t, u, u_{k}\right): u \in\left[\underline{x}^{a}(t), \bar{x}^{a}(t)\right], u_{k} \in\left[\underline{k}^{a}, \bar{k}^{a}\right]\right]
\end{gathered}
$$

where $\left.k^{a}=\left[\underline{k}^{a}, \bar{k}^{a}\right]\right]$. Then $x:[0, \infty) \rightarrow E^{1}$ is a solution of (2) using the Seikkala derivative and $x_{0}, k \in E^{1}$ if

$$
\begin{aligned}
& \left(\underline{x}^{a}\right)^{\prime}(t)=\min f\left(t, u, u_{k}\right): u \in\left[\underline{x}^{a}(t), \bar{x}^{a}(t)\right], u_{k} \in\left[\underline{k}^{a}, \bar{k}^{a}\right], \underline{x}^{a}(0)=\underline{x}_{0}^{a}, \\
& \left(\bar{x}^{a}\right)^{\prime}(t)=\max f\left(t, u, u_{k}\right): u \in\left[\underline{x}^{a}(t), \bar{x}^{a}(t)\right], u_{k} \in\left[\underline{k}^{a}, \bar{k}^{a}\right], \underline{x}^{a}(0)=\underline{x}_{0}^{a},
\end{aligned}
$$

for all $t \in[0, \infty)$ and $a \in[0,1]$.

\section{Leapfrog Method}

The most familiar and elementary method for approximating solutions of an initial value problem is Eulers Method. Eulers Method approximates the derivative in the form of $y^{\prime}=f(t, y), y\left(t_{0}\right)=y_{0}, y \in R^{d}$ by a finite difference quotient $y^{\prime} \approx(y(t+h)-y(t)) / h$. We shall usually discretize the independent variable in equal increments:

$$
t_{n+1}=t_{n}+h, \quad n=0,1, \ldots, t_{0} .
$$

Henceforth we focus on the scalar case, $N=1$. Rearranging the difference quotient gives us the corresponding approximate values of the dependent variable:

$$
y_{n+1}=y_{n}+h f\left(t_{n}, y_{n}\right), \quad n=0,1, \ldots, t_{0} .
$$

To obtain the leapfrog method, we discretize $t_{n}$ as in $t_{n+1}=t_{n}+h, n=$ $0,1, \ldots, t_{0}$, but we double the time interval, $h$, and write the midpoint approximation

$$
y(t+h)-y(t) \approx h y^{\prime}\left(t+\frac{h}{2}\right)
$$

in the form

$$
y^{\prime}(t+h) \approx(y(t+2 h)-y(t)) / h
$$

and then discretize it as follows:

$$
y_{n+1}=y_{n-1}+2 h f\left(t_{n}, y_{n}\right), \quad n=0,1, \ldots, t_{0} .
$$


The leapfrog method is a linear $m=2$-step method, with $a_{0}=0, a_{1}=$ $1, b_{-1}=-1, b_{0}=2$ and $b_{1}=0$. It uses slopes evaluated at odd values of $n$ to advance the values at points at even values of $n$, and vice versa, reminiscent of the childrens game of the same name. For the same reason, there are multiple solutions of the leapfrog method with the same initial value $y=y_{0}$. This situation suggests a potential instability present in multistep methods, which must be addressed when we analyze themtwo values, $y_{0}$ and $y_{1}$, are required to initialize solutions of $y_{n+1}=y_{n-1}+2 h f\left(t_{n}, y_{n}\right), n=0,1, \ldots, t_{0}$ uniquely, but the analytical problem $y^{\prime}=f(t, y), y\left(t_{0}\right)=y_{0}, y \in R^{d}$ only provides one. Also for this reason, one-step methods are used to initialize multistep methods.

\section{The Hybrid Fuzzy Differential Systems}

In this section, we study the fuzzy initial value problem for a hybrid fuzzy differential systems.

$$
x^{\prime}(t)=f\left(t, x(t), \lambda_{k} x\left(t_{k}\right)\right), \quad t \in\left[t_{k}, t_{k+1}\right], \quad x\left(t_{k}\right)=x_{t_{k}},
$$

where $x$ denotes Seikkala differentiation, $0 \leq t_{0}<t_{1}<\ldots<t_{k}<\ldots, t_{k} \rightarrow \infty$, $f \in C\left[R^{+} \times E^{1} \times E^{1}, E^{1}\right], \lambda_{k} \in C\left[E^{1}, E^{1}\right]$. To be specific the system look like

$$
x^{\prime}(t)=\left\{\begin{array}{l}
x_{0}^{\prime}(t)=f\left(t, x_{0}(t), \lambda_{0} x\left(t_{0}\right)\right), x_{0}\left(t_{0}\right)=x_{0}, t_{0} \leq t \leq t_{1}, \\
x_{1}^{\prime}(t)=f\left(t, x_{1}(t), \lambda_{1} x\left(t_{1}\right)\right), x_{1}\left(t_{1}\right)=x_{1}, t_{1} \leq t \leq t_{2}, \\
\cdots \\
x_{k}^{\prime}(t)=f\left(t, x_{k}(t), \lambda_{k} x\left(t_{k}\right)\right), x_{k}\left(t_{k}\right)=x_{k}, t_{k} \leq t \leq t_{k+1}, \\
\cdots
\end{array}\right.
$$

Assuming that the existence and uniqueness of solution of (3) hold for each $\left[t_{k}, t_{k+1}\right]$, by the solution of (4) we mean the following function:

$$
x(t)=x\left(t, t_{0}, x_{0}\right)\left\{\begin{array}{l}
x_{0}(t), t_{0} \leq t \leq t_{1} \\
x_{1}(t), t_{1} \leq t \leq t_{2} \\
\cdots \\
x_{k}(t), t_{k} \leq t \leq t_{k+1} \\
\cdots
\end{array}\right.
$$

We note that the solution of (5) are piecewise differentiable in each interval for $t \in\left[t_{k}, t_{k+1}\right]$ for a fixed $x_{k} \in E^{1}$ and $k=0,1,2, \ldots$ 
Using a representation of fuzzy numbers studied by Goestschel and Woxman [6] and $\mathrm{Wu}$ and $\mathrm{Ma}$ [23], we may represent $x \in E^{1}$ by a pair of functions $(\underline{x}(r), \bar{x}(r)), 0 \leq r \leq 1$, such that:

(i) $(\underline{x}(r)$, is bounded, left continuous, and non decreasing,

(ii) $\bar{x}(r)$ is bounded, left continuous, and non increasing, and

(iii) $(\underline{x}(r) \leq \bar{x}(r)), 0 \leq r \leq 1$.

For example, $u \in E^{1}$ given in (1) is represented by $(\underline{u}(r), \bar{u} r)=(0.75+$ $0.25 r, 1.5-0.5 r), 0 \leq r \leq 1$, which is similar to $[u]^{a}$ given by $(2)$.

Therefore we may replace (5) by an equivalent system

$$
\left\{\begin{array}{l}
\underline{x}^{\prime}(t)=\underline{f}\left(t, x, \lambda_{k} x\left(t_{k}\right)\right) \equiv F_{k}(t, \underline{x}, \bar{x}),\left(\underline{x}\left(t_{k}\right)=\bar{x}_{k}\right), \\
\underline{x}(t)=\bar{f}\left(t, x, \lambda_{k} x\left(t_{k}\right)\right) \equiv G_{k}(t, \underline{x}, \bar{x}),\left(\underline{x}\left(t_{k}\right)=\bar{x}_{k}\right),
\end{array}\right.
$$

which possesses a unique solution $(\underline{x}, \bar{x})$ which is a fuzzy function. That is for each $t$, the pair $[\underline{x}(t ; r), \bar{x}(t ; r)]$ is a fuzzy number, where $\underline{x}(t ; r), \bar{x}(t ; r)$ are respectively the solutions of the parametric form given by

$$
\left\{\begin{array}{l}
\underline{x^{\prime}}(t)=F_{k}(t, \underline{x}(t ; r), \bar{x}(t, r)), \underline{x}\left(t_{k} ; r\right)=\underline{x}_{k}(r), \\
\underline{x^{\prime}}(t)=G_{k}(t, \underline{x}(t ; r), \bar{x}(t, r)), \underline{x}\left(t_{k} ; r\right)=\underline{x}_{k}(r),
\end{array}\right.
$$

for $r \in[0,1]$.

\section{Numerical Examples}

In this section, we solved the hybrid fuzzy initial value problems to show the efficiency and accuracy of the proposed methods.

Consider the following hybrid fuzzy IVP, [19]

$$
\left.\begin{array}{r}
x^{\prime}(t)=x(t)+m(t) \lambda_{k} x\left(t_{k}\right), t \in\left[t_{k}, t_{k+1}\right], t_{k}=k, k=0,1,2,3, \ldots \\
x(t, r)=\left[(0.75+0.25 r) e^{t},(1.125-0.125 r) e^{t}\right], 0 \leq r \leq 1,
\end{array}\right\}
$$

Where

$$
\begin{gathered}
m(t)= \begin{cases}2(t(\bmod 1)) & \text { if } t(\bmod 1) \leq 0.5 \\
2(1-t(\bmod 1)) & \text { if } t(\bmod 1)>0.5\end{cases} \\
\lambda_{k}(\mu)= \begin{cases}\hat{0} & \text { if } k=0 \\
\mu & \text { if } k=1,2, \ldots\end{cases}
\end{gathered}
$$


The hybrid fuzzy IVP (6) is equivalent to the following systems of fuzzy IVPs:

$$
\begin{gathered}
x_{0}^{\prime}(t)=x_{0}(t), t \in[0,1], \\
x(0 ; r)=\left[(0.75+0.25 r) e^{t},(1.125-0.125 r) e^{t}\right], \quad 0 \leq r \leq 1, \\
x_{i}^{\prime}(t)=x_{i}(t)+m(t) x_{i-1}(t), \quad t \in\left[t_{i}, t_{i+1}\right], \quad x_{i}(t)=x_{i-1}\left(t_{i}\right), \quad i=1,2, \ldots
\end{gathered}
$$

In (6) $x(t)+m(t) \lambda_{k} x\left(t_{k}\right)$ is continuous function of $t, x$ and $\lambda_{k} x\left(t_{k}\right)$. Therefore by Example 6.1 of Kaleva [8], for each $k=0,1,2, \ldots$ the fuzzy IVP

$$
\left.\begin{array}{l}
x^{\prime}(t)=x(t)+m(t) \lambda_{k} x\left(t_{k}\right), t \in\left[t_{k}, t_{k+1}\right], \quad t_{k}=k, \\
x\left(t_{k}\right)=x_{t_{K}}
\end{array}\right\}
$$

has a unique solution $\left[t_{k}, t_{k+1}\right]$. To numerically solve the hybrid fuzzy IVP (7) we applied the Leapfrog method for hybrid fuzzy differential equation with $\mathrm{N}$ $=2$ to obtain $y_{1,2}(r)$ approximating $x(2.0 ; r)$. The Exact and Approximate solutions by STHWS and Leapfrog method are compared and the absolute error were shown in Table 1. From the Table 1, shows that Leapfrog method approximate solutions have less error compare to STHWS method solutions [19] in the all the stages.

Table 1: Error calculations

\begin{tabular}{cccccc}
\hline & \multicolumn{2}{c}{ STHWS Error } & & \multicolumn{2}{c}{ Leapfrog Error } \\
\cline { 2 - 3 } \cline { 5 - 6 }$t$ & $Y_{1}\left(t_{i} ; r\right)$ & $Y_{2}\left(t_{i} ; r\right)$ & & $Y_{1}\left(t_{i} ; r\right)$ & $Y_{2}\left(t_{i} ; r\right)$ \\
\hline 0.1 & $1 \mathrm{E}-07$ & $1 \mathrm{E}-07$ & & $1.01 \mathrm{E}-09$ & $1.11 \mathrm{E}-09$ \\
0.2 & $2 \mathrm{E}-07$ & $2 \mathrm{E}-07$ & & $2.01 \mathrm{E}-09$ & $2.11 \mathrm{E}-09$ \\
0.3 & $3 \mathrm{E}-07$ & $3 \mathrm{E}-07$ & & $3.01 \mathrm{E}-09$ & $3.11 \mathrm{E}-09$ \\
0.4 & $4 \mathrm{E}-07$ & $4 \mathrm{E}-07$ & & $4.01 \mathrm{E}-09$ & $4.11 \mathrm{E}-09$ \\
0.5 & $5 \mathrm{E}-07$ & $5 \mathrm{E}-07$ & & $5.01 \mathrm{E}-09$ & $5.11 \mathrm{E}-09$ \\
0.6 & $6 \mathrm{E}-07$ & $6 \mathrm{E}-07$ & & $6.01 \mathrm{E}-09$ & $6.11 \mathrm{E}-09$ \\
0.7 & $7 \mathrm{E}-07$ & $7 \mathrm{E}-07$ & & $7.01 \mathrm{E}-09$ & $7.11 \mathrm{E}-09$ \\
0.8 & $8 \mathrm{E}-07$ & $8 \mathrm{E}-07$ & & $8.01 \mathrm{E}-09$ & $8.11 \mathrm{E}-09$ \\
0.9 & $9 \mathrm{E}-07$ & $9 \mathrm{E}-07$ & & $9.01 \mathrm{E}-09$ & $9.11 \mathrm{E}-09$ \\
1.0 & $1 \mathrm{E}-06$ & $1 \mathrm{E}-06$ & & $1.01 \mathrm{E}-08$ & $1.11 \mathrm{E}-08$ \\
\hline
\end{tabular}




\section{Conclusions}

The obtained results (Error calculations) of the hybrid fuzzy differential equations show that the Leapfrog method works well for finding the solutions. From the table 1, it can be observed that for most of the time intervals, the absolute error is less in the Leapfrog method when compared to the STHWS method discussed by Sekar et al. [19], which yields a small error, along with the exact solutions. The method can be implemented using a digital computer. It occupies less memory space and consumes less computer time than the method in [19]. Illustrative example was included to demonstrate the validity and applicability of the technique. Hence the Leapfrog method is more suitable for studying the hybrid fuzzy differential equations.

\section{Acknowledgments}

The authors gratefully acknowledge the Dr. A. Murugesan, Assistant Professor, Department of Mathematics, Government Arts College (Autonomous), Cherry Road, Salem - 636 007, for his encouragement and support. The authors also thank Dr. S. Mehar Banu, Assistant Professor, Department of Mathematics, Government Arts College for Women, Salem - 636 008, Tamil Nadu, India, for her kind help and suggestions.

\section{References}

[1] O. Akin, T. Khaniyev, O. Oruç, I.B. Turksen, An algorithm for the solution of second order fuzzy initial value problems, Expert Systems with Applications, 40 (2013), 953-957.

[2] A. Bencsik, B. Bede, J. Tar, and J. Fodor. Fuzzy differential equations in modeling hydraulic differential servo cylinders. Proceedings of the 3rd Romanian-Hungarian Joint Symposium on Applied Computational Intelligence (SACI 2006), Timisoara, Romania, 2006.

[3] S. S. L. Chang and L. A. Zadeh. On fuzzy mapping and control. IEEE Transactions on Systems, Man, and Cybernetics, 2:30-34, 1972.

[4] D. Dubois and H. Prade. Towards fuzzy differential calculus. III. Differentiation. Fuzzy Sets and Systems, 8:225-233, 1982. 
[5] D. N. Georgiou, J. J. Nieto, and R. Rodriguez-Lopez. Initial value problems for higher-order fuzzy differential equations. Nonlinear Analysis: Theory, Methods and Applications, 63:587-600, 2005.

[6] R. Goetschel and D. Woxman. Elementary fuzzy calculus. Fuzzy Sets and Systems, 18:31-43, 1986.

[7] M. Guo, X. Xue, and R. Li. The oscillation of delay differential inclusions and fuzzy bio dynamics models. Mathematical and Computer Modeling, 37:651-658, 2003.

[8] M. Guo, X. Xue, and R. Li. Impulsive functional differential inclusions and fuzzy population models. Fuzzy Sets and Systems, 138: 601-615, 2003.

[9] O. Kaleva. Fuzzy differential equations. Fuzzy Sets and Systems, 24:301$317,1987$.

[10] O. Kaleva. The Cauchy problem for fuzzy differential equations. Fuzzy Sets and Systems, 35:389-396, 1990.

[11] S. Karunanithi, S. Chakravarthy and S. Sekar. Comparison of Leapfrog and single term Haar wavelet series method to solve the second order linear system with singular-A. Journal of Mathematical and Computational Sciences, 4:804-816, 2014.

[12] S. Karunanithi, S. Chakravarthy and S. Sekar. A Study on Second-Order Linear Singular Systems using Leapfrog Method. International Journal of Scientific and Engineering Research, 5:747-750, 2014.

[13] A. Khastan, F. Bahrami, and K. Ivaz. New results on multiple solutions for nth-order fuzzy differential equations under generalized differentiability. Boundary Value Problems, 2009:Article ID 395714, 2009.

[14] V. Lakshmikantham and R. Mohapatra, Theory of Fuzzy Differential Equations and Inclusions, Taylor and Francis, London, 2003.

[15] M. L. Puri and D. A. Ralescu. Differentials of fuzzy functions. Journal of Mathematical Analysis and Applications, 91:552-558, 1983.

[16] M. L. Puri and D. A. Ralescu. Fuzzy random variables. Journal of Mathematical Analysis and Applications, 114:409-422, 1986.

[17] M. Radstrom. An embedding theorem for space of convex sets. Proc. Amer. Math. Soc., 3:165-169, 1952. 
[18] S. Sekar and K. Prabhavathi. Numerical solution of first order linear fuzzy differential equations using Leapfrog method. IOSR Journal of Mathematics, 10:07-12, 2014.

[19] S. Sekar and S. Senthilkumar. Numerical Solution for Hybrid Fuzzy Systems by Single Term Haar Wavelet Series Technique. International Journal of Mathematical Archive 4:23-29, 2013.

[20] S. Sekar and S. Senthilkumar. A Study on Second-Order Fuzzy Differential Equations using STHWS Method. International Journal of Scientific and Engineering Research, 5:2111-2114, 2014.

[21] S. Sekar and M. Vijayarakavan. Numerical Investigation of first order linear Singular Systems using Leapfrog Method. International Journal of Mathematics Trends and Technology, 12:89-93, 2014.

[22] S. Seikkala. On the fuzzy initial value problem. Fuzzy Sets and Systems, 24:319-330, 1987.

[23] C. X. Wu and M. Ma. Embedding problem of fuzzy number space Part I. Fuzzy Sets and Systems, 44:33-38, 1991. 